\section{Modeling genetic complexity in vitro}

\section{By Tim Fulmer, Senior Writer}

Harvard researchers believe they have developed a method for generating in vitro models of highly complex genetic diseases, which are difficult to model with standard genetic knockout techniques. ${ }^{1}$ With proof of principle in a monogenic diabetes model, the researchers now plan to generate models of more complex genetic diseases and hope to use those models to identify new therapeutic targets and pathways.

Over the past decade, genomewide association (GWA) studies have helped identify genetic variation that influences susceptibility to and progression of genetic diseases. However, the development of models that incorporate such genetic variation has lagged.

As a result, single-gene knockout or knock-in techniques are still the dominant methods, even though GWA studies have shown that many genetic diseases are driven by variation in multiple genes that potentially influence a variety of cellular pathways.

Rather than attempt to engineer that complex genetic variation into a nonhuman cell line or animal model, a research team led by Stuart Schreiber and Stanley Shaw took a different tack and focused on patientderived cells as preclinical disease models.

The clear advantage is that patient-derived cells typically contain all the genetic complexity that underlies a disease and therefore should not require additional genetic engineering to model that condition.

Shaw is a professor of medicine at Harvard Medical School, a member of the Massachusetts General Hospital Center for Systems Biology and Cardiovascular Research Center and an affiliate of the Broad Institute of MIT and Harvard. Schreiber is a professor of chemistry and chemical biology at Harvard University and a core member of the Broad Institute.

Given the complexity involved in directly using patient-derived cells, the Harvard team needed to address two key problems: how to isolate patient cells that can serve as models of any particular genetic disease and how to probe those cells to uncover new therapeutic targets and disease mechanisms.

The researchers chose lymphoblast cell lines (LCLs) because such cells are readily accessible from the peripheral circulation and are often available from patient cohorts that were subjects of GWA studies. Moreover, work by other labs has suggested that LCLs can model both hematological and nonhematological disorders, such as hyperinsulinemia and Huntington's disease $(\mathrm{HD}){ }^{2,3}$
To serve as probes, the Harvard researchers chose libraries of compounds with known targets and mechanisms. Thus, if a given compound altered cellular metabolism, it would be clear what target or pathway the compound was modulating.

\section{Keep it complicated}

With cells and probes in hand, the researchers set out to get proof of principle for their approach in diabetes, a disease that has been an area of focus in Schreiber's and Shaw's labs for more than a decade.

First, their team obtained LCLs from patients with maturity onset diabetes of the young type 1 (MODY1), a severe form of disease that is caused by a mutation in the nuclear hormone receptor hepatocyte nuclear factor $4 \alpha$ (HNF4A; TCF).

Next, the researchers treated those lymphoblasts with 3,973 previously characterized small molecules. They looked for classes of compounds that significantly altered cellular ATP content, a marker of cellular energy homeostasis that is affected by metabolic disorders like diabetes.

Among 141 different compound classes, those that had the most significant effects on ATP levels in HNF4A-mutant cells compared with in wild-type cells included certain subsets of fatty acids $\left(p=6.1 \times 10^{-3}\right)$, potassium-sparing diuretics $(p=0.015), \mathrm{HMG}$ CoA reductase inhibitors $\left(p=7.9 \times 10^{-3}\right)$ and glucocorticoids $(p=0.028)$.

Finally, to confirm that measuring ATP levels in lymphoblasts was indeed a good surrogate marker for diabetes, the researchers looked at the best compound classes in cultured pancreatic $\beta$ cells that were deficient in HNF4A. They found that just over half of the compound classes had similar effects on ATP levels in these cells.

Several of the molecules also increased $\beta$ cell insulin secretion in the presence of high glucose, a cellular readout that is directly relevant to diabetes therapies.

The authors concluded: "Our data suggest that functional connections relevant to $\beta$-cell physiology can be elucidated from smallmolecule-gene interactions in a collection of patient-derived LCLs..."

They also pointed out that none of the compounds identified by the lymphoblast screen had been studied before in association with MODY1, "and none were approved for diabetes-related indications."

The findings were published in the Proceedings of the National Academy of Sciences.

Shaw told SciBX that the researchers now want to look at undisclosed genetic diseases, including autoimmune and inflammatory disorders.

"We believe our approach to creating cell culture models of genetic diseases could incorporate other patient-derived cell types, not only lymphoblasts. The choice of cell type for the model will be a balance of accessibility to the researcher and disease-relevant physiology," he said.

Shaw said the findings are not covered by any patent applications.

Fulmer, T. SciBX 4(1); doi:10.1038/scibx.2011.4

Published online Jan. 6, 2011 


\section{REFERENCES}

1. Shaw, S.Y. et al. Proc. Natl. Acad. Sci. USA;

published online Dec. 23, 2010; doi:10.1073/pnas.1016789108

Contact: Stuart L. Schreiber, Harvard University, Cambridge, Mass.

e-mail: stuart_schreiber@harvard.edu

Contact: Stanley Y. Shaw, Massachusetts General Hospital,

Boston, Mass.

e-mail: shaw.stanley@mgh.harvard.edu
2. MacMullen, C. et al. J. Clin. Endocrinol. Metab. 86, 1782-1787 (2001)

3. Seong, I.S. et al. Hum. Mol. Genet. 14, 2871-2880 (2005)

COMPANIES AND INSTITUTIONS MENTIONED

Broad Institute of MIT and Harvard, Cambridge, Mass.

Harvard Medical School, Boston, Mass.

Harvard University, Cambridge, Mass.

Massachusetts General Hospital, Boston, Mass. 